\title{
CSN8 is a key regulator in hypoxia-induced epithelial-mesenchymal transition and dormancy of colorectal cancer cells
}

\author{
Songwen $\mathrm{Ju}^{1 *+}$, Feng Wang ${ }^{2 \dagger}$, Yirong Wang $^{1}$ and Songguang Ju $3,4,5^{*}$ (D)
}

\begin{abstract}
Hypoxic stress plays a pivotal role in cancer progression; however, how hypoxia drives tumors to become more aggressive or metastatic and adaptive to adverse environmental stress is still poorly understood. In this study, we revealed that CSN8 might be a key regulatory switch controlling hypoxia-induced malignant tumor progression. We demonstrated that the expression of CSN8 increased significantly in colorectal cancerous tissues, which was correlated with lymph node metastasis and predicted poor patient survival. CSN8 overexpression induces the epithelial-mesenchymal transition (EMT) process in colorectal cancer cells, increasing migration and invasion. CSN8 overexpression arrested cell proliferation, upregulated key dormancy marker (NR2F1, DEC2, p27) and hypoxia response genes (HIF-1a, GLUT1), and dramatically enhanced survival under hypoxia, serum deprivation, or chemodrug 5-fluorouracil treatment conditions. In particular, silenced CSN8 blocks the EMT and dormancy processes induced by the hypoxia of $1 \% \mathrm{O}_{2}$ in vitro and undermines the adaptive capacity of colorectal cancer cells in vivo. The further study showed that CSN8 regulated EMT and dormancy partly by activating the HIF-1a signaling pathway, which increased HIF-1a mRNA expression by activating NF-KB and stabilized the HIF-1a protein via HIF-1a de-ubiquitination. Taken together, CSN8 endows primary colorectal cancer cells with highly aggressive/metastatic and adaptive capacities through regulating both EMT and dormancy induced by hypoxia. CSN8 could serve as a novel prognostic biomarker for colorectal cancer and would be an ideal target of disseminated dormant cell elimination and tumor metastasis, recurrence, and chemoresistance prevention.
\end{abstract}

Keywords: CSN8, Hypoxia, Epithelial-mesenchymal transition, Dormancy, Colorectal cancer

\section{Main text}

Cancer fatalities result from metastasis and therapy resistance, with both processes depending on signals from the tumor microenvironment [1]. Hypoxia is a common feature of the cancer microenvironment and is strongly associated with invasion, metastasis, resistance to

\footnotetext{
* Correspondence: Jusongwen@aliyun.com; Songguang_ju@hotmail.com ${ }^{\dagger}$ Songwen Ju and Feng Wang contributed equally to this work

'Central Laboratory, Affiliated Suzhou Hospital of Nanjing Medical University, Suzhou Municipal Hospital, Suzhou 215002, Jiangsu Province, China ${ }^{3}$ Department of Immunology, School of Biology and Basic Medical Sciences, Medical College, Soochow University, Suzhou 215123, Jiangsu Province, China

Full list of author information is available at the end of the article
}

therapy, and poor clinical outcomes. Hypoxia plays a crucial role in triggering the epithelial-mesenchymal transition (EMT) by regulating hypoxia-inducible factors (HIFs) [2]. EMT represents a key step toward cancer cell migration from the primary tumor to the distant organs and, ultimately, to disseminated tumor cells (DTCs) [3]. Furthermore, hypoxia has been shown to induce molecular changes within cancer cells to facilitate dormancy, which is a state of "temporary mitotic arrest" [4]. Accumulating evidences indicate that dormancy is essential for cancer cell survival in "hostile" microenvironments at distant sites, as cells become resistant to cancer therapy and evade attack by immune cells $[4,5]$.

C C The Author(s). 2020 Open Access This article is licensed under a Creative Commons Attribution 4.0 International License, which permits use, sharing, adaptation, distribution and reproduction in any medium or format, as long as you give appropriate credit to the original author(s) and the source, provide a link to the Creative Commons licence, and indicate if changes were made. The images or other third party material in this article are included in the article's Creative Commons licence, unless indicated otherwise in a credit line to the material. If material is not included in the article's Creative Commons licence and your intended use is not permitted by statutory regulation or exceeds the permitted use, you will need to obtain permission directly from the copyright holder. To view a copy of this licence, visit http://creativecommons.org/licenses/by/4.0/ The Creative Commons Public Domain Dedication waiver (http://creativecommons.org/publicdomain/zero/1.0/) applies to the data made available in this article, unless otherwise stated in a credit line to the data. 
However, the underlying mechanism through which cancer cells undergo EMT, dormancy progression, and evade apoptosis/necrosis under hypoxic environments still remains to be elucidated.

The COP9 signalosome (CSN) is an evolutionarily conserved protein complex composed of 8 subunits (CSN1-CSN8) in higher eukaryotes, and plays an important role in ubiquitin-mediated protein degradation [6]. Previous reports indicated that the subunit of CSN, such as CSN6, is involved the malignant process of cancer [7]. CSN8, the smallest and least conserved subunit among the eight subunits of CSN, is essential for peripheral $\mathrm{T}$ cell homeostasis and cardiomyocytes survival [6]. However, the role of CSN8 in cancer biology is still unclear. Here, we demonstrated that the expression of CSN8 is upregulated in colorectal cancer (CRC) tissues and correlates with lymph node metastasis and poor prognosis. CSN8 serves as a key regulator that controls EMT and dormancy induced by hypoxia, partly through modulating the HIF- $1 \alpha$ signaling pathway, endowing CRC cells with stronger invasion and metastatic abilities, as well as highly adaptive capacities in response to environmental stress.

\section{Results and discussion}

\section{CSN8 is upregulated in CRC tissues, and involved in the}

\section{EMT and dormancy of CRC cells}

To investigate the expression of CSN8 in CRC tissues, we conducted immunohistochemistry (IHC) staining for CSN8 on a CRC tissue microarray (product number: HCol-Ade 180Sur-14) containing 90 pairs of CRC specimens and the corresponding tumor-adjacent tissues. Three pairs of tumor and tumor-adjacent tissues were excluded as they were severely broken, and the samples from the other $87 \mathrm{CRC}$ patients were further analyzed. CSN8 was dominantly expressed in the nucleus and weakly in the cytoplasm of CRC tissues and adjacent tissues (Fig. 1a). CSN8 was expressed at statistically significantly higher levels in the nucleus of CRC tissues, as compared to adjacent tissues (Fig. 1b). The high expression of CSN8 was found to be significantly associated with lymph node metastasis, tumor stage (Additional file 2: Table S1), and poor patient survival (Fig. 1c). The data from the other set of CRC tissue microarray (product number: HCol-Ade 180Sur-06) confirmed the above findings and indicated that there was no significant correlation between the expression of CSN8 and the status of mismatch repair (MMR) proteins (Additional File 3. Fig. S3; Additional File 2. Table S4).

Since EMT is a process that is associated with tumor metastasis and poor prognosis, we then clarified whether the high expression of CSN8 was involved in the EMT process. The serial sections of tissue microarrays by immunohistochemistry (IHC) staining revealed that CSN8 expression was negatively correlated with EMT maker E-Cadherin expression (Fig. 1d; Additional file 2: Table S2). Accordingly, CMV-drivenCSN8 overexpression suppresses the expression of ECadherin, whereas it enhances the expression of mesenchymal marker genes (N-Cadherin and Vimentin), the EMT transcription factor (EMT-TF) Slug, and matrix metallopeptidases (MMP2, MMP3 and MMP9) in CRC cell lines HCT116 and DLD-1 (Fig. 1e and l). Wound-healing assays showed that CSN8 overexpressed HCT116, and that DLD-1 cells healed the wound much faster than did the control cells (Fig. If and g). Transwell migration assay (Fig. $1 \mathrm{~h}$ and i) and the matrigel invasion assay (Fig. $1 \mathrm{j}$ and $\mathrm{k}$ ) showed that the over-expression of CSN8 increased the migration and invasion of CRC cell lines.

Since dormancy, a state of permanent growth arrest, endowed cancer cells' strong resistance and tolerance to environmental stress, we discussed the relationship between CSN8 and the dormancy of CRC cells. As expected, the CCK-8 assay revealed that the overexpression of CSN8 significantly inhibited the proliferation of HCT116 and DLD-1 cells compared to the control cells (Fig. 1m). CSN8 overexpressed HCT116 and DLD-1 cells, which were found to have higher vitality under hypoxia, serum deprivation conditions, or 5-FU treatment compared to the control cells (Figs. 1n-p). The CSN8-overexpressed HCT116 and DLD-1 cells expressed higher levels of dormant markers (NR2F1, DEC2, p27), hypoxia genes (HIF-1 $\alpha$, GLUT1), and lower levels of Ki67 (Fig. 1q and r), which indicates that CSN8 may be involved in regulating hypoxia-induced dormancy. The overexpression of CSN8 can suppress the expression of c-Myc (Fig. 1q and r). Proto-oncogene c-Myc regulates cell proliferation, cell-cycle progression, and tumor growth. Further, c-Myc inactivation results in tumor dormancy. Suppression of c-Myc protein levels contributes to cancer cell survival under limited oxygen and glucose [8]. Therefore, the downregulation of c-Myc induced by CSN8 might be an important factor contributing to the induction of a dormant state and apoptosis resistance, as well as the promotion of survival under oxygen- and serum-deprived conditions. It was reported that pluripotency-associated genes increased in dormant tumor cells [9]. We also found that CSN8 overexpression could enhance the expression of pluripotency-associated genes (SOX2 and SOX9; Fig. 1r). In addition, a significant increase in the anti-apoptosis gene $B C L-2$ and immunosuppressive gene $P D-L 1$ expression (Fig. 1r) were detected in CSN8-overexpressed cells, which might be associated with enhanced tolerance to environmental stress and 


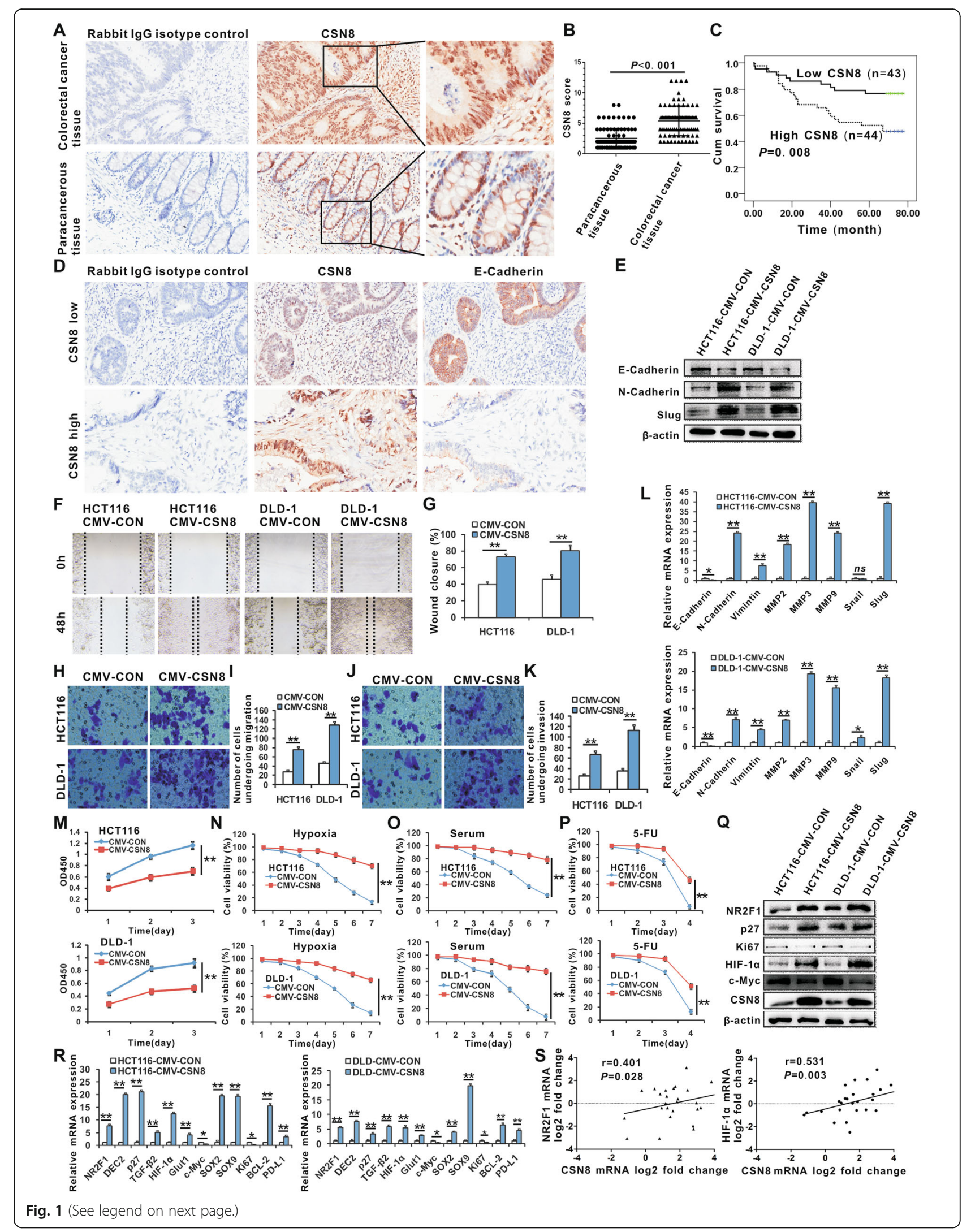




\begin{abstract}
(See figure on previous page.)
Fig. 1 CSN8 expression is upregulated in CRC tissues, and is involved in the EMT and dormancy of CRC cells. a Tissue microarray assays analyzed the expression of CSN8 in CRC tissues. Representative immunohistochemistry images showed the expression and subcellular distribution of CSN8 in tumor tissues or tumor-adjacent tissues. b Nuclear CSN8 expression is higher in tumor tissues than in tumor-adjacent tissues. c Kaplan-Meier survival analysis was conducted according to the CSN8 levels in CRC patients (log-rank test). $\mathbf{d}$ Representative immunohistochemistry images show the expression of CSN8 and E-Cadherin in tumor tissues. e Western-blot analysis of protein levels of E-Cadherin, N-Cadherin, and Slug in CSN8-overexpressed CRC cells and control cells. $\mathbf{f}, \mathbf{g}$ Representative scratch-wound images showing the healing ability of CSN8-overexpressed CRC cells and control cells. $\mathbf{h}, \mathbf{i}$ The migration ability of CSN8-overexpressed CRC cells and control cells was determined by Transwell migration assay. $\mathbf{j}, \mathbf{k}$ The invasive ability of CSN8-overexpressed CRC cells and control cells was analyzed by Matrigel invasion assay. I Real-time PCR was used to analyze the mRNA expression of EMT-associated genes. $\mathbf{m}$ CCK-8 assay analyzed the proliferation activity of CSN8-overexpressed CRC cells and control cells. $\mathbf{n}-\mathbf{p}$ Trypan blue exclusion assay was used to analyze the cell viability of CSN8-overexpressed CRC cells and control cells cultured under $20 \% \mathrm{O}_{2}$ or $1 \% \mathrm{O}_{2}$, serum deprivation, or $5-\mathrm{FU}(20 \mathrm{\mu g} / \mathrm{mL})$ conditions. q Western blot analysis of the protein levels of dormancyassociated genes. $\mathbf{r}$ Real-time PCR was used to analyze the mRNA expression of dormancy-associated genes. $\mathbf{s}$ Human CRC tissue CDNA arrays showing changes in CSN8, NR2F1, or HIF-1a transcript expression. Data are presented as the mean \pm standard deviation. ${ }^{*} P<0.05{ }^{* * *} P<0.01$; $n s, P \geq 0.05$
\end{abstract}

immunoescape. The CRC tissue cDNA array showed that the mRNA expression of CSN8 is positively correlated with that of NR2F1 and HIF-1 $\alpha$ (Fig. 1s), which suggests that CSN8 participates in regulating hypoxia-induced dormancy. The silencing of CSN8 further verified that CSN8 could act as an important mediator or regulator of EMT and dormancy (Additional file 3: Figs. S1).

\section{CSN8 is essential for inducing EMT and dormancy under hypoxic environments and for developing stress resistance}

To mimic the hypoxic and inflammatory factors of the tumor microenvironment, we cultured HCT116 and DLD- 1 cells with $1 \%$ oxygen or with the addition of tumor necrosis factor (TNF)- $\alpha$ or interleukin (IL)$1 \beta$. We found that CSN8 expression was upregulated under the stimulation of hypoxia, TNF- $\alpha$, or IL- $1 \beta$ by real-time polymerase chain reaction (PCR) assay (Fig. 2a). To determine whether CSN8 mediated EMT and dormancy induced by hypoxia, we cultured CSN8-silenced HCT116 and DLD-1 cells under 1\% oxygen conditions. The results showed that hypoxia failed to drive these two CSN8-silenced CRC cell lines to undergo EMT processes, whereas the control cells obviously demonstrated morphological changes from the more tightly associated epithelial phenotype to the loosely connected, elongated, mesenchymal phenotype (Fig. 2b). Apoptosis assay using Annexin $\mathrm{V} /$ propidium iodide (PI) staining and flow cytometry showed that hypoxia significantly induced apoptosis in CSN8-silenced HCT116 and DLD-1 cells when compared to the control cells (Fig. 2c). Real-time PCR analysis revealed that silencing CSN8 inhibited the E/N-Cadherin switch and attenuated increased levels of Slug, NR2F1, p27, HIF-1 $\alpha$, and BCL-2 under hypoxic conditions (Fig. 2d). These results indicate that CSN8 is essential in the EMT and dormancy process in CRC cells, while also contributing to the evasion of apoptosis/necrosis under hypoxic environments. In tumor-bearing mouse models, the tumor formation and tumor growth of CSN8-silenced HCT116 and DLD-1 cells had decreased in comparison with the control cells (Additional file 3: Figs. S2). Silencing of CSN8 might block the dormant process in vivo for CRC cells and impair their adaptive ability in the tumor microenvironment.

As nuclear factor $\mathrm{\kappa B}(\mathrm{NF}-\mathrm{\kappa B})$ is a direct modulator of HIF- $1 \alpha$ expression, we used the luciferase reporter assay to monitor NF- $\mathrm{kB}$ activation. CSN8 overexpression significantly enhanced NF- $\mathrm{kB}$ transcriptional activation (Fig. 2e). DHMEQ, an NF- $\mathrm{kB}$ inhibitor, could suppress the HIF- $1 \alpha$ mRNA expression induced by CSN8 overexpression (Fig. 2f). HIF-1 $\alpha$ is a short-lived protein and is ubiquitinated and degraded through the von Hippel-Lindau protein (pVHL)-E3 ubiquitin ligase pathway at normoxia [2]. CSN is reportedly associated with deubiquitination activity [6]. The ubiquitination assay showed that CSN8 overexpression could decrease HIF- $1 \alpha$ ubiquitination (Fig. $2 \mathrm{~g})$. Therefore, these results indicated that CSN8 induced the expression of HIF-1 $\alpha$ through NF- $\mathrm{KB}$ activation and HIF- $1 \alpha$ deubiquitination. Inhibition of HIF- $1 \alpha$ by LW6, a HIF- $1 \alpha$ inhibitor, resulted in the suppression of the CSN8-mediated E/N-Cadherin switch and the expression of Slug, NR2F1, DEC2, and p27 (Fig. 2h). Hence, CSN8 regulates EMT and dormancy partially by activating the HIF- $1 \alpha$ pathway. HIF- $1 \alpha$ can bind directly to Sp1, resulting in c-Myc displacement from Sp1 complexes and inhibiting cMyc transcriptional activity. However, it does not alter c-Myc mRNA and protein levels [10]. Thus, downregulation of c-Myc expression induced by CSN8 may occur independently of the increase in HIF- $1 \alpha$ expression, which is an important factor that contributes to dormancy as well. 


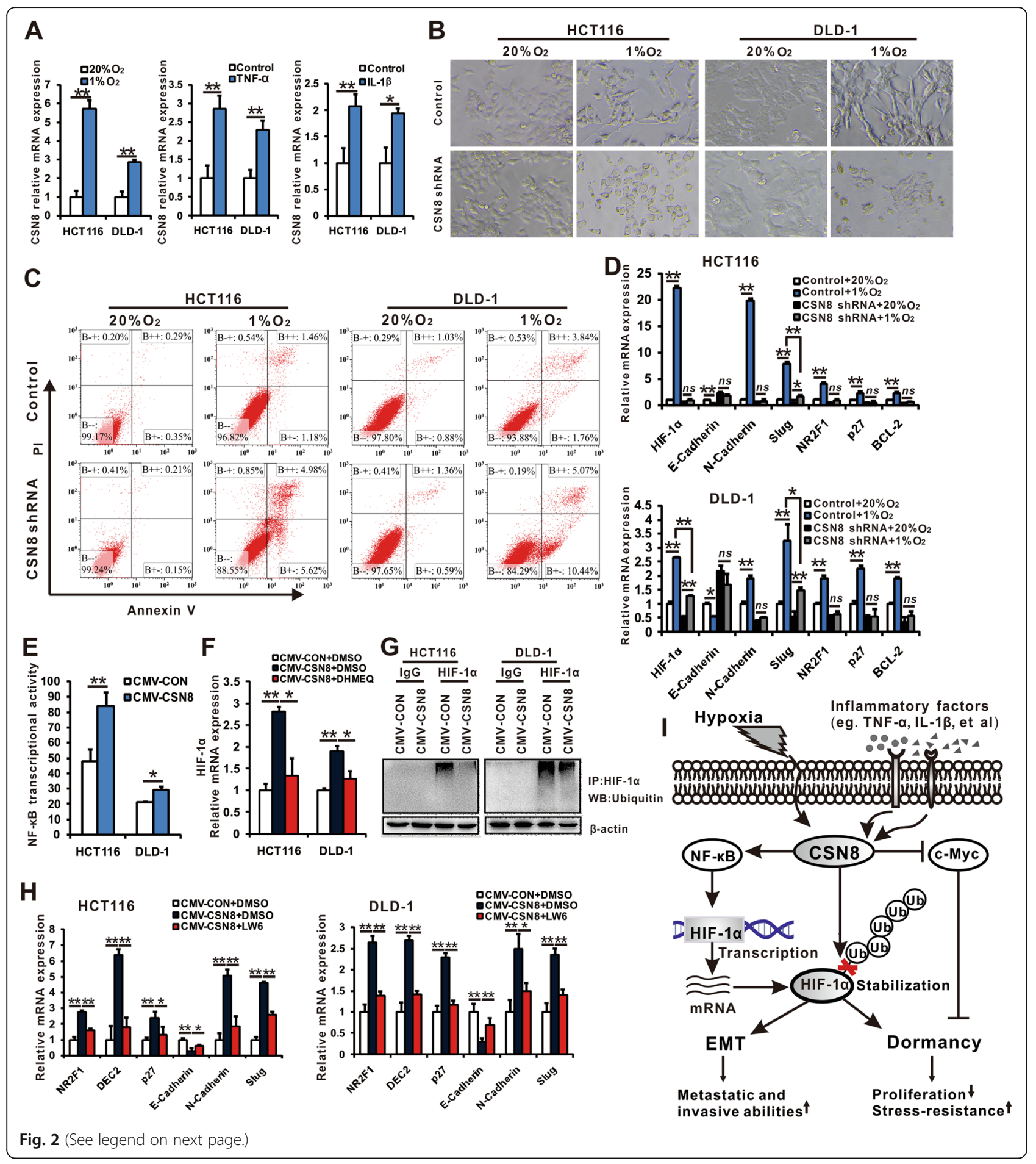


(See figure on previous page.)

Fig. 2 CSN8 is essential for inducing EMT and dormancy under hypoxic environments, and for developing stress resistance. a HCT116 and DLD-1 cells were cultured under $20 \% \mathrm{O}_{2}$ or $1 \% \mathrm{O}_{2}$ conditions, or treated with TNF-a $(20 \mathrm{ng} / \mathrm{mL})$ or IL-1 $\beta(20 \mathrm{ng} / \mathrm{mL})$ for $24 \mathrm{~h}$, and then real-time PCR was used to analyze CSN8 mRNA expression. b CSN8-silenced CRC cells and control cells were cultured under $20 \% \mathrm{O}_{2}$ or $1 \% \mathrm{O}_{2}$ conditions for another $48 \mathrm{~h}$. Photographs were taken using a phase-contrast microscope. c Annexin V-FITC/PI staining assay was used to analyze the apoptotic and necrotic cells. $\mathbf{d}$ Real-time PCR was used to analyze the mRNA expression of the hypoxic response, EMT, and dormancy-related genes. e CSN8-overexpressed CRC cells and control cells were transfected with NF-KB dual-luciferase plasmid or control plasmid. After being transfected for $48 \mathrm{~h}$, luciferase activity was detected with the dual-luciferase reporter assay system. $\mathbf{f}$ CSN8-overexpressed CRC cells were treated with NF-KB inhibitor DHMEQ $(25 \mu \mathrm{M})$ or DMSO solvent control for $24 \mathrm{~h}$, and then real-time PCR was used to analyze the HIF-1a mRNA levels. $\mathbf{g}$ Western-blot analysis of the HIF-1a-immunoprecipitated lysates with the anti-ubiquitin antibody. $\mathbf{h}$ CSN8-overexpressed CRC cells were treated with the HIF-1a inhibitor LW6 $(20 \mu \mathrm{M})$ or DMSO solvent control for $24 \mathrm{~h}$, and then real-time PCR was used to analyze the mRNA levels of EMT and dormancyrelated genes. $\mathbf{i}$ The model depicts the pivotal role of CSN8 in the EMT and dormancy process of CRC cells under the hypoxic microenvironment. ${ }^{*} P<0.05 ;{ }^{* *} P<0.01 ; n s, P \geq 0.05$

\section{Conclusions}

Our findings revealed that CSN8 might be a key regulatory molecule that controls the hypoxia-induced EMT and dormancy process, which endows CRC cells with highly aggressive, metastatic and adaptive capacities (Fig. 2i). CSN8 would be an ideal target of disseminated dormant cell elimination and tumor metastasis, recurrence and chemoresistance prevention.

\section{Supplementary Information}

The online version contains supplementary material available at https://doi. org/10.1186/s12943-020-01285-4.

\section{Additional file 1:. Supplementary materials and methods.}

Additional file 2: Table S1. Correlation between the expression of CSN8 and the clinicopathological features of CRC patients. Table S2. Correlation between the expression of CSN8 and E-Cadherin. Table S3. Primer sequences used for quantitative Real-Time PCR. Table S4. Correlation between the expression of CSN8 and the clinicopathological features of CRC patients from a parallel study.

Additional file 3 Figure S1. Silencing CSN8 reverses EMT and the dormancy of CRC cells. Figure S2. Silencing CSN8 undermines the survival of CRC cells in vivo. Figure S3. A parallel tissue microarray assay confirmed CSN8 expression is upregulated in CRC tissues and correlated to poor outcome.

\section{Abbreviations}

CRC: Colorectal cancer; 5-Fu: 5-fluorouracil; EMT: Epithelial-mesenchymal transition; HIFs: Hypoxia inducible factors; DTCs: Disseminated tumor cells; MMPs: Matrix metalloproteinases; HNSCC: Head and neck squamous cell carcinoma; CSN: COP9 signalosome; IHC: Immunohistochemistry; NF$\mathrm{kB}$ : Nuclear factor $\mathrm{kB}$

\section{Acknowledgements}

The authors would like to thank Dr. Christina Gallucci of Journal Prep for the English-language editing.

\section{Authors' contributions}

SWJ and SGJ conceived the project, designed the experiments, interpreted the data, and wrote the manuscript. SWJ, FW, YRW and SGJ conducted the experiments and collected the data. All authors read and approved the final Manuscript.

\section{Funding}

This work was supported by grants from National Natural Science Foundation of China (81373149, 31370887), Nature Science Foundation of Jiangsu Province (BK20151195), Fund of Medical Youth Talent Project of Jiangsu Province (QNRC2016241), Fund of Collaborative Innovation Center of Clinical Immunology between Soochow University and Sihong People's
Hospital, Priority Academic Program Development of Jiangsu Higher Education Institutions (PAPD).

\section{Availability of data and materials}

All the data obtained and/or analyzed during the current study were available from the corresponding authors on reasonable request.

\section{Ethics approval and consent to participate}

All protocols for this study was reviewed and approved by Suzhou Municipal Hospital Institutional Ethics Committee and all animal protocols were approved by the Institutional Laboratory Animal Care and Use Committee at Soochow University (Suzhou, People's Republic of China).

\section{Consent for publication}

The content of this manuscript has not been previously published and is not under consideration for publication elsewhere.

\section{Competing interests}

The authors declare that they have no competing interests.

\section{Author details}

${ }^{1}$ Central Laboratory, Affiliated Suzhou Hospital of Nanjing Medical University, Suzhou Municipal Hospital, Suzhou 215002, Jiangsu Province, China. ${ }^{2}$ Department of Pathology, Affiliated Suzhou Hospital of Nanjing Medical University, Suzhou Municipal Hospital, Suzhou 215002, Jiangsu Province, China. ${ }^{3}$ Department of Immunology, School of Biology and Basic Medical Sciences, Medical College, Soochow University, Suzhou 215123, Jiangsu Province, China. ${ }^{4}$ Medical Biotechnology Institute, Soochow University, Suzhou 215123, Jiangsu Province, China. ${ }^{5}$ Collaborative Innovation Center of Clinical Immunology between Soochow University and Sihong People's Hospital, Soochow University, Suzhou 215123, Jiangsu Province, China.

Received: 20 August 2020 Accepted: 20 November 2020 Published online: 01 December 2020

\section{References}

1. Haeger A, Alexander S, Vullings M, Kaiser FM, Veelken C, Flucke U, Koehl GE, Hirschberg M, Flentje M, Hoffman RM. Collective cancer invasion forms an integrin-dependent radioresistant niche. J Exp Med. 2020;217:e20181184.

2. Rankin EB, Giaccia AJ. Hypoxic control of metastasis. Science. 2016;352: 175-80.

3. Brabletz T, Kalluri R, Nieto MA, Weinberg RA. EMT in cancer. Nat Rev Cancer. 2018;18:128-34.

4. Fluegen $G$, Avivar-Valderas A, Wang Y, Padgen MR, Williams JK, Nobre AR, Calvo V, Cheung JF, Bravo-Cordero JJ, Entenberg D, et al. Phenotypic heterogeneity of disseminated tumour cells is preset by primary tumour hypoxic microenvironments. Nat Cell Biol. 2017;19:120-32.

5. Phan TG, Croucher PI. The dormant cancer cell life cycle. Nat Rev Cancer. 2020:1-14.

6. Milic J, Tian Y, Bernhagen J. Role of the COP9 Signalosome (CSN) in cardiovascular diseases. Biomolecules. 2019;9:217.

7. Fang L, Lu W, Choi HH, Yeung SC, Tung JY, Hsiao CD, Fuentes-Mattei E, Menter D, Chen C, Wang L, et al. ERK2-dependent phosphorylation of 
CSN6 is critical in colorectal Cancer development. Cancer Cell. 2015;28: 183-97.

8. Okuyama H, Endo H, Akashika T, Kato K, Inoue M. Downregulation of c-MYC protein levels contributes to Cancer cell survival under dual deficiency of oxygen and glucose. Cancer Res. 2010;70:10213-23.

9. Sosa MS, Parikh F, Maia AG, Estrada Y, Bosch A, Bragado P, Ekpin E, George A, Zheng Y, Lam HM, et al. NR2F1 controls tumour cell dormancy via SOX9- and RARbeta-driven quiescence programmes. Nat Commun. 2015;6:6170.

10. Koshiji M, Kageyama Y, Pete EA, Horikawa I, Barrett JC, Huang LE. HIF-1a induces cell cycle arrest by functionally counteracting Myc. EMBO J. 2004;23: 1949-56.

\section{Publisher's Note}

Springer Nature remains neutral with regard to jurisdictional claims in published maps and institutional affiliations.

- fast, convenient online submission

- thorough peer review by experienced researchers in your field

- rapid publication on acceptance

- support for research data, including large and complex data types

- gold Open Access which fosters wider collaboration and increased citations

- maximum visibility for your research: over $100 \mathrm{M}$ website views per year

At BMC, research is always in progress. 